\title{
OMAE2006-92253
}

\section{HYDRODYNAMIC WAVE LOADING ON OFFSHORE STRUCTURES SIMULATED BY A TWO-PHASE FLOW MODEL}

\author{
Rik Wemmenhove \\ Department of Mathematics \\ University of Groningen \\ PO Box 800, 9700 AV Groningen \\ The Netherlands \\ Email: r.wemmenhove@math.rug.nl
}

\author{
Erwin Loots \\ Department of Mathematics \\ University of Groningen \\ PO Box 800, 9700 AV Groningen \\ The Netherlands
}

\author{
Arthur E.P. Veldman \\ Department of Mathematics \\ University of Groningen \\ PO Box 800, 9700 AV Groningen \\ The Netherlands
}

\begin{abstract}
The numerical simulation of hydrodynamic wave loading on different types of offshore structures is important to predict forces on and water motion around these structures. This paper presents a numerical study of the effects of two-phase flow on an offshore structure subject to breaking waves.
\end{abstract}

The details of the numerical model, an improved Volume Of Fluid (iVOF) method, are presented in the paper. The program has been developed initially to study the sloshing of liquid fuel in satellites. This micro-gravity environment requires a very accurate and robust description of the free surface. Later, the numerical model has been used for calculations of green water loading and the analysis of anti-roll and sloshing tanks, including the coupling with ship motions.

The model has been extended recently to take two-phase flow effects into account. Two-phase flow effects are particularly important near the free surface, where loads on offshore structures strongly depend on the interaction between different phases like air and water. Entrapment of air pockets and entrainment of bubble clouds have a cushioning effect on breaking wave impacts.

The velocity field around the interface of air and water, being continuous across the free surface, requires special attention. By using a newly-developed gravity-consistent discretisation, spurious velocities at the free surface are prevented.

Thus far, the second air phase has been treated as incompressible. Taking compressibility effects into account requires a pressure-density relation for grid cells containing air. The expansion and compression of air pockets is considered as an adiabatic process.

The numerical model is validated on several test cases. In this paper special attention will be paid to the impact of a breaking wave over a sloping bottom on a fixed offshore structure.

\section{NOMENCLATURE}

$\begin{array}{lll}\mathrm{dt} & {[s]} & \text { time step } \\ \mathrm{F} & {[\mathrm{N}]} & \text { body force } \\ \mathrm{g} & {\left[\mathrm{ms}^{-2}\right]} & \text { gravity vector } \\ \mathrm{h} & {[\mathrm{m}]} & \text { grid cell spacing } \\ \mathrm{n} & {[\mathrm{s}]} & \text { time level } \\ \mathrm{p} & {[\mathrm{Pa}]} & \text { pressure } \\ \mathrm{u} & {\left[\mathrm{ms}^{-1}\right]} & \text { velocity } \\ \mathrm{V} & {\left[\mathrm{m}^{3}\right]} & \text { volume } \\ \gamma & {[-]} & \text { adiabatic coefficient } \\ \mu & {\left[\mathrm{kgm}^{-1} \mathrm{~s}^{-1}\right]} & \text { dynamic viscosity } \\ \rho & {\left[\mathrm{kgm}^{-3}\right]} & \text { density }\end{array}$

\section{INTRODUCTION}

During violent weather conditions offshore structures are subject to different types of wave impact. The impacting waves are complex mixtures of water and air, the properties of the mixture having a serious effect on velocity fields around and presure levels on offshore structures. During wave impact on vertical 
walls, there is experimental evidence [1] that peak pressure levels decrease due to the presence of air in the water, however the duration of the wave impact may increase. The two-phase

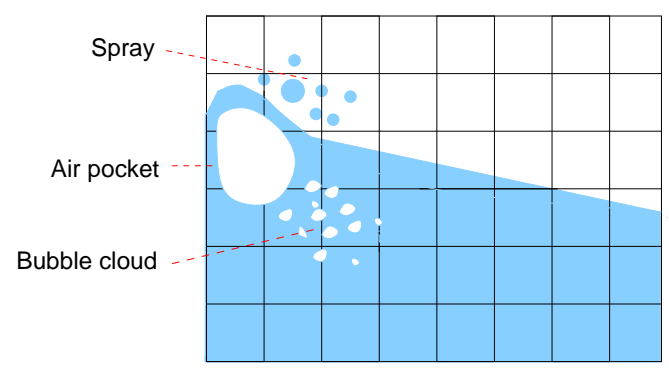

Figure 1. Two-phase phenomena at a breaking wave

flow effects mainly occur in the vicinity of the free surface, see figure 1. Spray is of minor relevance for determining pressure loads, but air pockets and bubbles seriously affect hydrodynamic forces.

Simulations of hydrodynamic wave loading occur mainly by one-phase models, considering only the water phase. Most models focus on specific aspects of free surface flows, such as wave impact of aerated flow on walls or the velocity field under breaking waves.

As soon as flow conditions are getting more violent, using a two-phase flow model is strongly recommendable. However, the small spatial and temporal scales of the air pockets and bubbles are a serious problem. Typical spatial scales in offshore domains are of the order of hundreds of meters, while air pockets and bubbles hardly reach sizes of 1 meter. So, keeping track of the air phase in large-scale offshore problem is a challenging task, even when using powerful computers.

Existing two-phase models focus either on single bubbles or on quite regular waves. Scardovelli [9] gives a nice overview of existing two-phase flow models. During the last few years, there is much progress in the simulation of two-phase flow [2], [7], [11]. Furthermore, computer power is getting cheaper, increasing the possibilities to simulate large problems. However, keeping track of very small individual bubbles is still extremely difficult, and problems like spurious velocities at the free surface are not prevented by using a faster computer.

In the present paper a two-phase numerical model is presented. The model is aimed to simulate a wide range of offshore problems. In this paper the model is tested on a breaking wave over a sloping bottom.

The first section of this paper describes the physical and mathematical framework of the numerical model. Compared with a one-phase numerical model, the implementation of a two-phase model requires the registration of more flow variables in the whole domain. After the physical-mathematical model, the details of the numerical model, i.e. an improved Volume Of Fluid (iVOF) method are presented. Particular attention is paid to a proper representation of the free surface. By using a correct density treatment, spurious velocities at the free surface are prevented. Before showing the results of a test case, different aspects of adding compressibility to the gas phase in the two-phase model are discussed. Finally, the results of a simulation with breaking wave impact over slopes with different steepnesses are presented and discussed. Comparisons are made between one-phase simulations and two-phase simulations of this numerical model and of a BEM method.

\section{Governing equations}

For each point in the domain the fluid motion is governed by the continuity equation and the momentum equation:

$$
\begin{array}{r}
\frac{\partial \rho}{\partial t}+\nabla \cdot(\rho u)=0 \\
\frac{\partial(\rho u)}{\partial t}+\underbrace{\nabla \cdot(\rho u u)}_{\text {convection }}+\nabla p+\underbrace{\nabla \cdot(\mu \nabla u)}_{\text {diffusion }}+\rho F=0
\end{array}
$$

with $F$ an external body force like gravity, velocity $u$, pressure $p$, density $\rho$ and dynamical viscosity $\mu$.

For incompressible flow, the velocity field is divergence-free, reducing the continuity equation to

$$
\nabla \cdot u=0
$$

The continuity equation and momentum equation are, after semidiscretisation in time, combined to the pressure Poisson equation to compute the new pressure value and velocity field. This pressure equation is shown in the next section.

\section{NUMERICAL MODEL iVOF algorithm}

The advection of water and air in the current model is based on the Volume Of Fluid (VOF) algorithm as developed by Hirt and Nichols [6].

As long as a one-phase approach was used for the model, the incompressible Navier-Stokes equations were solved with a free-surface condition on the free boundary. The VOF function $F_{S}$ (with values between 0 and 1 ) determined whether or not the flow field in a grid cell was calculated.

This is in contrast with the two-phase approach, where the 
air-water interface is no longer considered as a free surface, although the interface is still reconstructed using the VOF algorithm. Numerically important is now the density jump across the air-water interface, as the density can increase or decrease a factor 1000 , imposing a challenge to the numerical stability of the model. The liquid fraction $F_{S}$ is now used to calculate the aggregated density in a grid cell.

The numerical model has been implemented in a 3D VOF Navier-Stokes solver called CoMFLOW. Compared with the original VOF algorithm, a local height function improves the treatment of the free surface. The program has been developed initially by the University of Groningen to study the sloshing of liquid fuel in satellites [4]. This micro-gravity environment requires a very accurate and robust description of the free surface. In close cooperation with MARIN (Maritime Research Institute Netherlands), this methodology was later extended to the calculation of green water loading on a fixed bow deck. Also anti-roll tanks, including the coupling with ship motions, were investigated. Furthermore, the entry of a wedge in a fluid was studied as part of the RuG-MARIN cooperation, as well as the wave impact loads on fixed structures [7].

\section{Discretisation}

The discretisation of the Navier-Stokes equations is done on a staggered Cartesian grid, which means that the pressure is set in the cell centres and the velocity components in the middle of the cell faces between two grid cells.

For incompressible flow conditions, the Navier-Stokes equations, as given by eq.(1) and eq.(2), are discretised in time according to the explicit first order Forward Euler method:

$$
\begin{array}{r}
\nabla \cdot u^{n+1}=0 \\
\frac{u^{n+1}-u^{n}}{d t}+\nabla \cdot(u u)^{n}+\frac{1}{\rho^{n}} \nabla p^{n+1} \\
+\frac{1}{\rho^{n}} \nabla\left(\mu^{n} \nabla u^{n}\right)+F^{n}=0
\end{array}
$$

with $n$ the old time level and $n+1$ the new time level.

Now, the divergence of the momentum equation is taken. The term $\nabla \cdot u^{n+1}$ is isolated and inserted in the momentum equation. This results in the pressure Poisson equation:

$\nabla \cdot\left(\frac{1}{\rho^{n}} \nabla p^{n+1}\right)=\frac{1}{d t}\left(\nabla \cdot u^{n}\right)-\nabla^{2}\left(u^{n} u^{n}\right)-\nabla\left(\frac{1}{\rho} \nabla(\mu \nabla u)\right)-\nabla \cdot F^{n}$

After this equation has been solved for the pressure, the new velocity field $u^{n+1}$ is found by inserting this new pressure field in the momentum equation (5). The discretisation of the compressible equations follows below.

\section{Cell labeling}

To distinguish between the different characters of grid cells, the cells are labeled. The variable $F_{b}$ describes the fraction of a grid cell open for fluid, while the variable $F_{s}$ describes the fraction of a grid cell filled with the liquid phase. The Navier-Stokes equations are solved in grid cells containing at least one of the fluids.

Every grid cell is given a label to distinguish between boundary, air and fluid. Two classes of labeling exist: geometry cell labels and fluid cell labels.

The geometry labeling at each time step divides the cells into three classes:

F(low) cells: All cells with $F_{b} \geq 0$

$\mathbf{B}$ (oundary) cells: All cells adjacent to a F-cell

(e) $\mathbf{X}$ (ternal) cells: All remaining cells

The free surface cell labeling is a subdivision of the $\mathbf{F}$ cells, consisting of 3 subclasses:

E(mpty) cells: All cells with $F_{s}=0$

S(urface) cells: All cells adjacent to an E-cell

F'(luid) cells: All remaining F-cells
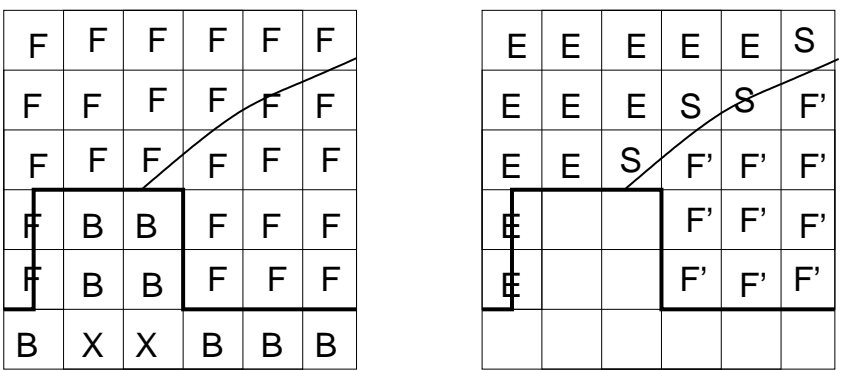

Figure 2. Geometry cell labeling (left) and free-surface cell labeling for wave impact on a rectangular container

Figure 2 shows an example of geometry cell labeling and free-surface cell labeling for wave impact from the right on a rectangular container.

The labeling system is retained in the two-phase model. In the one-phase model the empty cells were truly empty as no computations were performed there, while boundary conditions for the free surface were prescribed around surface cells. In the two-phase model, however, the empty cells contain the fluid with the smallest density. The Navier-Stokes equations are also solved in these cells. 


\section{Density treatment}

Special attention should be given to the discretisation of the density. Like the pressure, the density is a scalar variable, originally located at the center of a grid cell. Physically, it has only two values: the density of water and the density of air.

According to the solution method, the velocity field at the new time level is acquired by adding a term to the old velocity field (see equation (5)). Since the velocity field is highly continuous between the old and new time level, this term, $\nabla p / \rho$, should also be smooth. Since the pressures are located in cell centres, the term $\nabla p$ has to be calculated in a control volume located between two cell centers. The density value $\rho$ should hold - and be defined properly - in the control volume located which is located between the cell centres.

Consider the situation in figure 3 . The free surface dividing water (below) and air (above) is in this case a straight line with a slope of $\frac{1}{2}$. We need for both the horizontal and vertical direction a proper discretisation of the term $\nabla p / \rho$. The suggested control volumes for these terms are depicted with dashed lines, between cells $\mathrm{A}$ and $\mathrm{F}$ (vertical), and $\mathrm{E}$ and $\mathrm{A}$ (horizontal). As the pressure values are registrated at the circles, the discretization can be straighforward: $\left.\frac{1}{\rho} \frac{\partial p}{\partial x}\right|_{E A} \approx \frac{1}{h_{x}} \frac{\left(P_{A}-P_{E}\right)}{\rho_{E A}}$. Clearly, $\rho_{E A}=1$. However, it is not clear beforehand what $\rho_{A B}$ could be. Choices like 1 ("cell centres A and B both in air"), $125.875\left(=\frac{1}{2}\left(1 \cdot 1+\frac{1}{4} \cdot 1000+\frac{3}{4} \cdot 1\right)\right)$ or even 63.4375 $\left(=1 \cdot 1+\frac{1}{8} \cdot 1000+\frac{7}{8} \cdot 1\right)$ can be defended.

Does it matter? In fact, quite a lot. Consider the situation above, and make it stationary by setting the gravity vector $g=$ $\left(g_{x}, g_{z}\right)^{T}=(5,-10)^{T}$. In that case, with all time derivatives and velocities zero, the momentum equations (2) are reduced to

$$
\frac{\partial p}{\partial x}=\rho_{x} g_{x}, \quad \frac{\partial p}{\partial z}=\rho_{z} g_{z}
$$

Concentrating on the four cells A,B,C and D, we take the simple approach of defining the densities between the cells as the average of the weighted cell averages. So cell averages are approximately $\rho_{A}=1, \quad \rho_{B}=250, \quad \rho_{C}=750$ and $\rho_{D}=1000$. Further we suppose the cells have unit size.

Integrating the pressure in clockwise direction from A to D (via B) gives

$$
\begin{array}{r}
P_{D}=P_{B}-h_{z} g_{z} \rho_{B D}=P_{A}+h_{x} g_{x} \rho_{A B}-h_{z} g_{z} \rho_{B D} \\
=P_{A}+5 \cdot 125+10 \cdot 625=P_{A}+6875,
\end{array}
$$

while integrating in counterclockwise direction (from $A$ to $D$ via $C)$ gives

$$
\begin{array}{r}
P_{D}=P_{C}+h_{x} g_{x} \rho_{C D}=P_{A}-h_{z} g_{z} \rho_{A C}+h_{x} g_{x} \rho_{C D} \\
=P_{A}+10 \cdot 375+5 \cdot 875=P_{A}+8125 .
\end{array}
$$

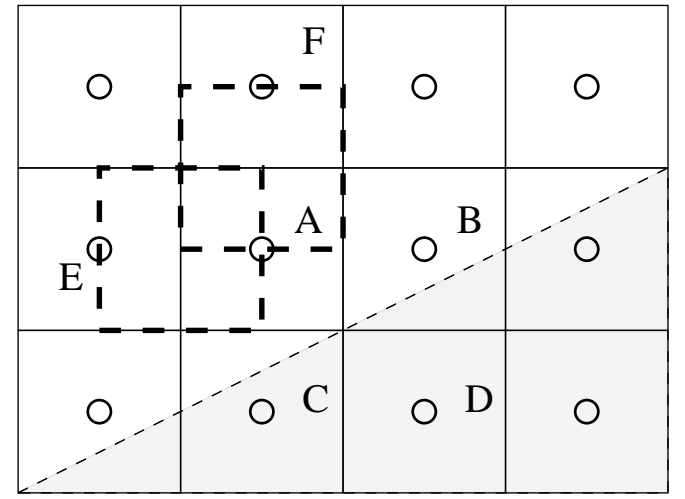

Figure 3. Averaging of density. The cells are numbered according to their position in the flow field.

This inconsistency (the contour integral not being zero) is immediately visible numerically: spurious velocities will occur around the free surface, see figure 5. So a more consistent density discretisation, which at least will cope correctly with stationary situations, is needed.

This very simple skewed-gravity example, however, provides a strategy for such a gravity-consistent discretisation. The pressure is known analytically: hydrostatic and 'aerostatic' in both phases, respectively. If we choose $p=0$ at the free surface then (see figure 4):

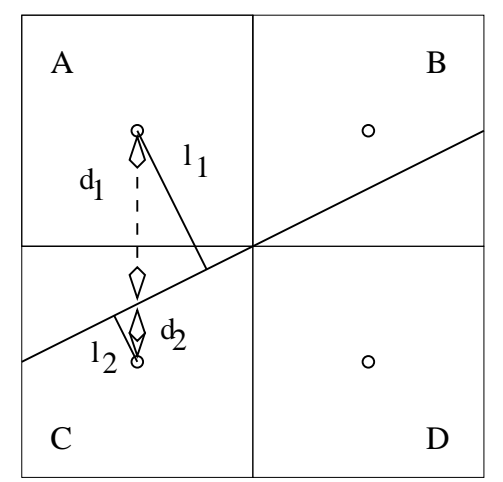

Figure 4. Gravity-consistent discretisation

$$
P_{A}=-l_{1} \rho_{1}|g|=d_{1} \cos \alpha \frac{g_{z}}{\cos \alpha}=d_{1} \rho_{1} g_{z}
$$

where $\alpha$ is the slope angle, and $P_{C}=l_{2} \rho_{2}|g|=-d_{2} \rho_{2} g_{z}$.

Together with $\frac{1}{\rho_{z}} \frac{\partial p}{\partial z}=g_{z}$, this gives $\rho_{A C}=\frac{1}{g_{z}} \frac{P_{A}-P_{C}}{d_{1}+d_{2}}=$ $\frac{d_{1} \rho_{1}+d_{2} \rho_{2}}{d_{1}+d_{2}}$. 
A similar construction gives simply $\rho_{A B}=\rho_{1}$, because both cell centres are in the fluid with density $\rho_{1}$. Using the local height function also used in the iVOF algorithm, the distances $d_{1}$ and $d_{2}$ can easily be computed.

In the given example, this leads to $\rho_{A B}=1, \rho_{A C} \approx$ $250, \rho_{B D} \approx 750$ and $\rho_{C D}=1000$, which gives a consistent density field and no spurious velocities.

Note that in this approach, the cell pressure was in some sense indentified with the pressure in the centre of the cell. This leads to the possibility of other ways of averaging the density, besides this 'cell-centered'-approach. Some of them are still under investigation.

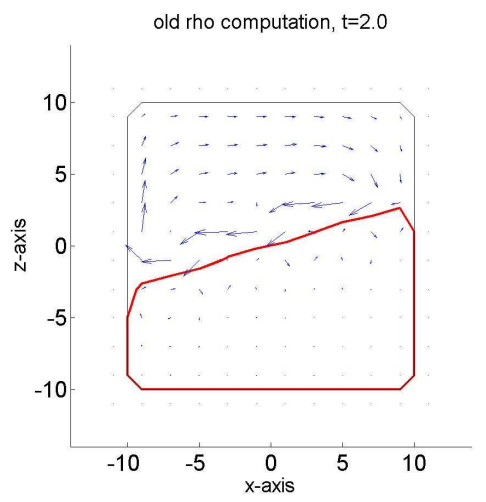

Figure 5. Spurious velocities due to simple averaging of density

\section{Extension to the modeling of compressible flow}

For compressible flow, the divergence-free condition of the velocity field, $\nabla \cdot u=0$, no longer holds, so we keep the semidiscretised form of the continuity equation (1):

$$
\frac{\rho^{n+1}-\rho^{n}}{d t}+u^{n} \nabla \rho^{n}+\rho^{n} \nabla \cdot u^{n+1}=0
$$

This equation is divided by the density and the term $\nabla \cdot u^{n+1}$ is substituted again by the divergence of the momentum equation. The acquired Poisson equation is similar to equation (6), but with two extra terms:

$$
\begin{array}{r}
\nabla \cdot\left(\frac{1}{\rho^{n}} \nabla p^{n+1}\right)=\frac{1}{d t}\left(\nabla \cdot u^{n}\right)-\nabla^{2}\left(u^{n} u^{n}\right)- \\
\nabla\left(\frac{1}{\rho} \nabla(\mu \nabla u)\right)-\nabla \cdot F^{n}+\frac{1}{d t \rho^{n}} \frac{\rho^{n+1}-\rho^{n}}{d t}+\frac{1}{d t} \frac{u^{n}}{\rho^{n}} \nabla \rho^{n}
\end{array}
$$

These extra terms are numerically dangerous because of the spatial and temporal derivatives of the density; at the free surface, the density can jump from 1 to 1000 . However, we can split the density in an incompressible liquid part $\rho_{l}$ and a compressible gas part $\rho_{g}$. Using the VOF function $F_{S}$, the cell density $\rho$ is given by $F_{s} \rho_{l}+\left(1-F_{s}\right) \rho_{g}$. Using $\frac{D F_{s}}{D t}=0$, the derivatives in equation (10) of the liquid part of the density reduce to $\frac{F_{s}}{d t \rho} \frac{D \rho_{l}}{D t}$, which is zero.

The remaining derivatives of the gas densities do not contain large jumps, as these derivatives are only determined by the compression and expansion of the gas phase. By the split up of the density into a liquid and gas part, the pressure Poisson equation (10) reduces to

$$
\begin{array}{r}
\nabla \cdot\left(\frac{1}{\rho^{n}} \nabla p^{n+1}\right)=\frac{1}{d t}\left(\nabla \cdot u^{n}\right)-\nabla^{2}\left(u^{n} u^{n}\right)- \\
\nabla\left(\frac{1}{\rho} \nabla(\mu \nabla u)\right)-\nabla \cdot F^{n}+\frac{1}{d t \rho^{n}} \frac{\rho_{g}^{n+1}-\rho_{g}^{n}}{d t}+\frac{1}{d t} \frac{u^{n}}{\rho_{g}^{n}} \nabla \rho^{n}
\end{array}
$$

The gas density at the new time level can be calculated by using the adiabatic pressure-density relation

$$
\frac{p^{n}}{p_{0}}=\left(\frac{\rho^{n}}{\rho_{0}}\right)^{\gamma},
$$

with $p_{0}$ and $\rho_{0}$ the reference pressure and density values.

These reference values need some consideration. As we are using an iterative solver, the reference pressure and density values can be taken from the previous timestep, so $\frac{p^{n+1}}{p^{n}}=\left(\frac{\rho^{n+1}}{\rho^{n}}\right)^{\gamma}$. The adiabatic coefficient $\gamma$ has a value of 1.4 for pure air.

\section{Testing compressible flow - falling water mass}

To give a simple example of compressible flow, consider the falling water mass as sketched in figure 6. This is essentially a 1D-situation, with gravity in vertical direction.

It provides an easy example of the differences between the one-phase computational model and the incompressible and compressible two-phase models. The ceiling of the column consists of an open boundary with Dirichlet condition $p=0$. In the three cases, the following wil happen:

one-phase model. The non-fluid part, i.e. the part which will not be labeled with E-cells, is not modeled: no equations are solved there. In the F(luid) cells, the full NavierStokes equations involve the gravity term $\rho_{\text {liq }} g$. The liquid will freely accelerate downward (fall) without a pressure inside the fluid cells until the bottom is reached. At the time step when the lowest cell is filled, it will be labeled an F-cell, because of the construction described above. This involves 


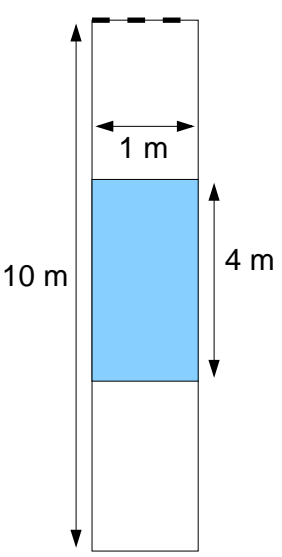

Figure 6. ID schematisation of a falling water mass

mass conservation, so the liquid stops falling and the pressure will be hydrostatically distributed immediately. The pressure gradient now exactly counteracts the gravity force. incompressible two-phase model This model involves solving the incompressible Navier-Stokes equations in both phases. Because of the incompressibility, the air below the liquid cannot move and a static situation is attained immediately. The pressure now increases linearly from the ceiling $(p=0)$ with a small slope for the air, a large slope in the liquid part and again a small slope in the lower air part.

compressible two-phase model. The air is now compressible. According to the adiabatic relationship $\rho_{2}=\rho_{1}\left(\frac{p_{2}}{p_{1}}\right)^{\frac{1}{\gamma}}$, the air volume $V_{\text {air }}$ under the liquid column will decrease until the pressure difference of the air above and below the liquid compensates for the downward force $g m_{\text {fluid }}$ while the relation $p V_{\text {air }}^{\gamma}=$ const remains valid. For example, with $\gamma=1, m_{l i q}=\rho_{l i q} V_{l i q}=4000$, the pressure under the liquid will eventually be $140 \mathrm{kPa}$ with a volume of $\frac{100}{140} V_{\text {air }, 0}$. The actual simulation indeed shows this behaviour (see figure 7).

\section{MODEL RESULTS: Breaking waves over a sloping bot- tom}

Breaking waves are good test cases to show the performance of a two-phase simulation in comparison with a one-phase simulation. The breaking waves in this test case are generated by a wave maker, being a model for a vessel generating bow waves or for the slamming of a ship hull on the ocean surface. The propagation, shoaling and breaking of waves over a gently sloping bottom are a challenge for numerical simulation [5]. Gently sloping bottoms are characteristic in shallow-water environments, where the hydrodynamics in the surf zone and near dikes are important.

Figure 8 shows the numerical domain. The simulation is

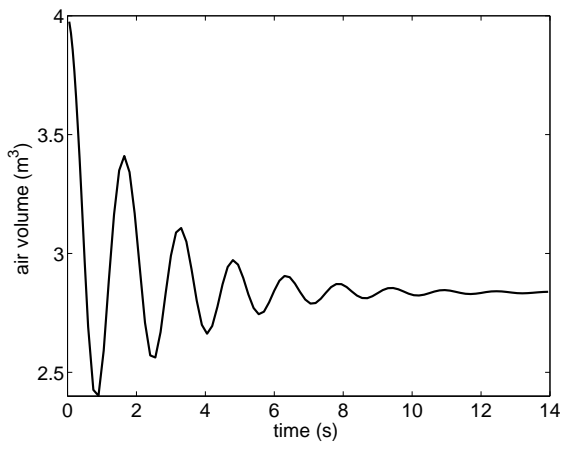

Figure 7. Pressure development in the air below a falling water mass for compressible flow
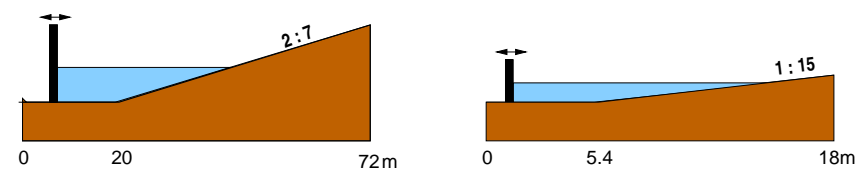

Figure 8. Geometry of the two test cases. The wave motion is induced by a wavemaker with a sinusoidal motion. Water depth $H_{0}=6 m$ for the steep $2: 7$ slope and $H_{0}=1 m$ for the mild $1: 15$ slope.

done in $2 \mathrm{D}$, as the flow effects in the third dimension are not important for interpreting the simulations. The wave maker on the left side of the domain, with a periodical oscillation in horizontal direction, creates solitary waves. The bottom and right boundaries of the domain are considered as solid boundaries, while the top boundary is considered as an outflow boundary for air. Using an outflow boundary condition at the boundary is necessary for two-phase simulations, as the air phase needs to "breathe" when the wave maker is pushing against it. The boundary condition at the top wall is the von Neumann boundary condition for both the pressure and velocity. Different slopes have been tested.

\section{Breaking waves on steep slope 2:7}

The wave runup on a steep slope or beach occurs over a short distance, associated with a strong deceleration of the wave. For this slope steepness, waves are characterized as "plunging" breakers. The undisturbed water depth $H_{0}=6 \mathrm{~m}$, while the wavemaker has an amplitude of $2.0 \mathrm{~m}$ and a frequency of $1 \mathrm{~s}^{-1}$. The simulations have been run with a grid of $144 \times 1 \times 144$ cells.

One-phase simulation The snapshots of the one-phase simulation in figure 9 show a relatively early breaking point, i.e. the point with a vertical wave front. An air pocket is trapped progressively around $x=32 \mathrm{~m}$. The trapped air pocket remains 

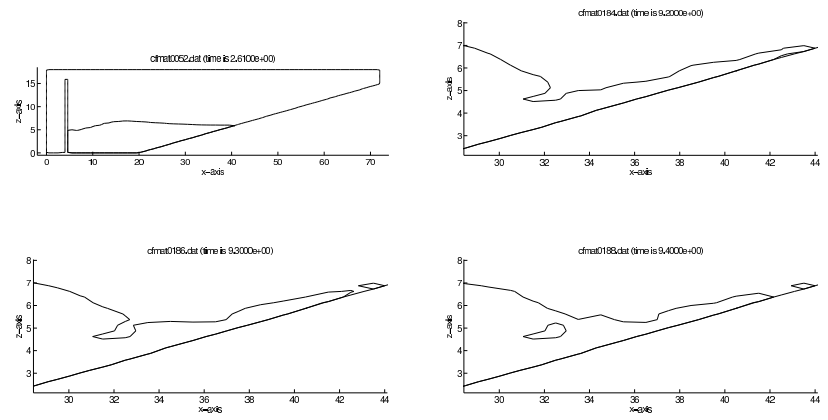

Figure 9. Snapshots of wave breaking for one-phase simulation and a steep 2:7 slope. The first picture shows the geometry long before wave breaking, the three other pictures show the wave front during and just after wave breaking.

present until the wave is flowing back in left direction. In this simulation the free surface is going through the air pocket at $t=$ $13.3 \mathrm{~s}$, dissolving the air pocket.

Two-phase simulation For the two-phase simulation the snapshots in figure 10 show entrapment of air as well. The breaking point of the wave is at $x=30 \mathrm{~m}$, see the upper figure. So wave breaking occurs later than for the one-phase simulation. This can be attributed to the viscosity in the air, increasing the energy dissipation during wave shoaling. The entrapment and closure of an air pocket under the wave front takes about $1 s$ from that moment. After closure of the air pocket, a breaker jet develops due to the splash-up of the wave (see the bottom left picture in figure 10). This breaker jet results again in subsequent entrapment of air pockets (bottom right picture in fig.10). This pattern of air entrapment has also been reported in Lachaume et al [8].

\section{Breaking waves on mild slope 1:15}

For the milder $1: 15$ slope, waves run up over a larger distance, as the waves are decelerated less strong. For this slope steepness, waves are characterized as collapsing breakers. The undisturbed water depth is now much smaller, $H_{0}=1 \mathrm{~m}$. The wavemaker has again an amplitude of $2.0 \mathrm{~m}$ and a frequency of $1 s^{-1}$. The simulations have been run with a grid of $180 \times 1 \times 100$ cells.

One-phase simulation and comparison The results can be compared with the simulations of Garzon et al [3]. Garzon et al used a similar geometry, but their model is a Boundary Element Method (BEM), a fully nonlinear potential model for incompressible, irrotational and inviscid flow. They distinguish the breaking point (location with a vertical wave front) and the
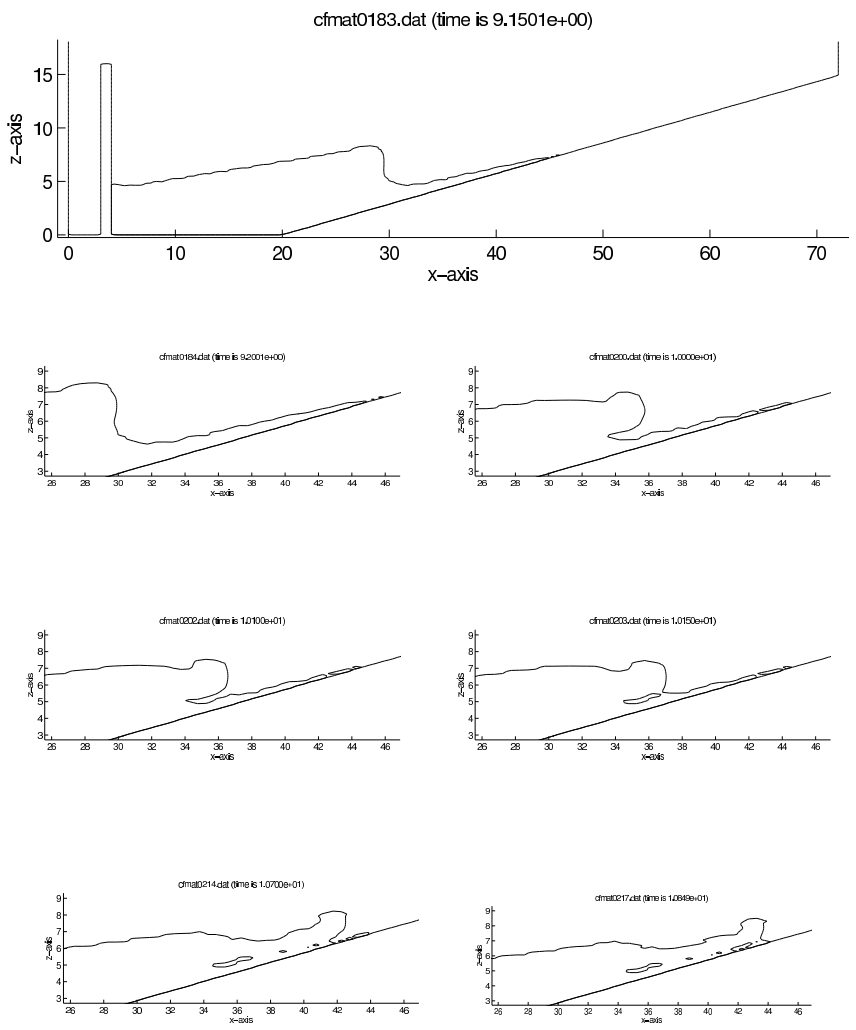

Figure 10. Snapshots of wave breaking for two-phase simulation and a steep 2:7 slope

\begin{tabular}{cllll}
\hline Model & $t_{b p}$ & $x_{b p}$ & $t_{e p}$ & $x_{e p}$ \\
\hline CoMFLOW 1-phase & 2.25 & 13.8 & 2.35 & 14.2 \\
Garzon et al [3] & 2.34 & 15.2 & 2.90 & 17.8 \\
\hline
\end{tabular}

Table 1. Breaking characteristics compared with Garzon et al [3]

end point (location with closure of an air pocket). For the mild slope geometry they show the following results:

The potential model of Garzon et al [3] shows a long period between the breaking point and the closure of the air pocket at the end point. This period is expected to be shorter in the physical reality. The breaking point in COMFLOW is earlier than in the simulation of Garzon et al, one of the reasons could be the viscosity (physical and numerical) that is included in CoMFLOW. The numerical results of this model will improve by refining the grid, as many grid cells are required in the $x$-direction to obtain 

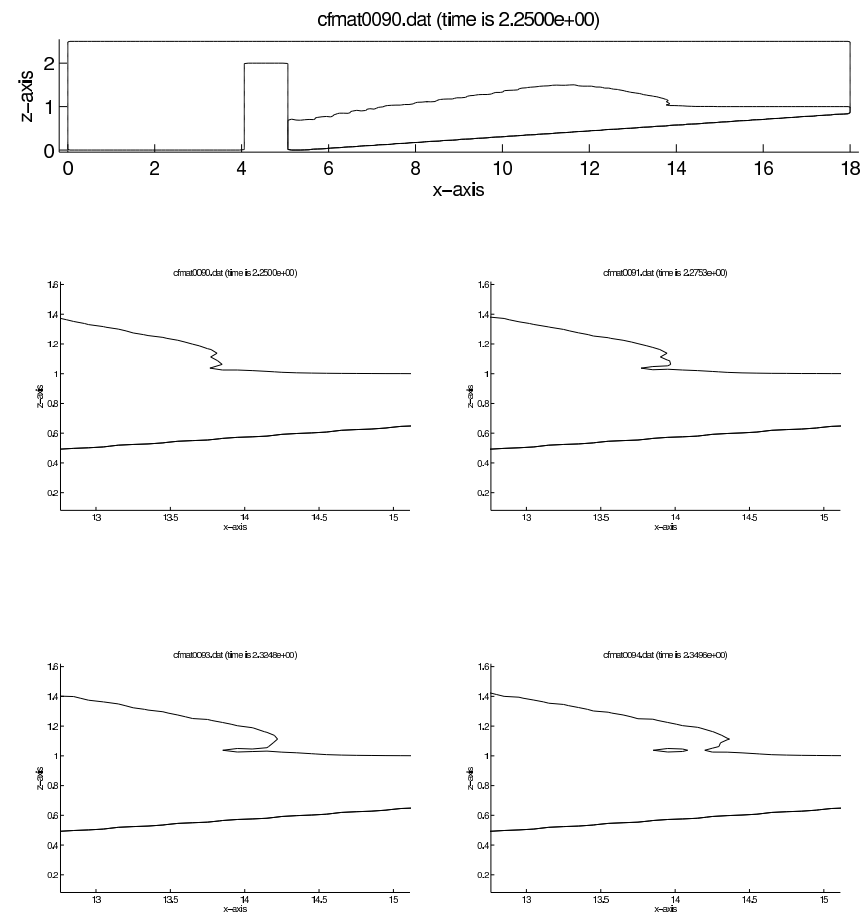

Figure 11. Snapshots of wave breaking for one-phase simulation and a mild 1:15 slope

accurate results.

Two-phase simulation Figure 12 shows some snapshots of the two-phase simulation for the wave runup on a mild $1: 15$ slope. The results could be compared with the one-phase simulation or with a BEM simulation, but the physics are different now as the viscosity of the air plays a significant role. Compared with the one-phase simulation, the propagating wave has again a greater effort to push away the viscous air. The result is that the first wave is not clearly breaking. However, the second wave generated by the wavemaker is breaking quite early.

The breaking point is already at $x=4.8 \mathrm{~m}$, while a significant air pocket is trapped around $x=9 m$ (fig.12(e)). The air pocket has a height of about 4 grid cells, containing a number of $E$-cells. In the one-phase simulation, this air pocket would remain on the same location (until the free surface crossed it), as the velocity field is not calculated in the air phase.

In this two-phase simulation, however, this air pocket is moving in right direction, parallel with the motion of the wave. The velocity field is shown to be continuous across the boundaries of the air pocket (see the velocity field in figure $12(\mathrm{~g})$ ).
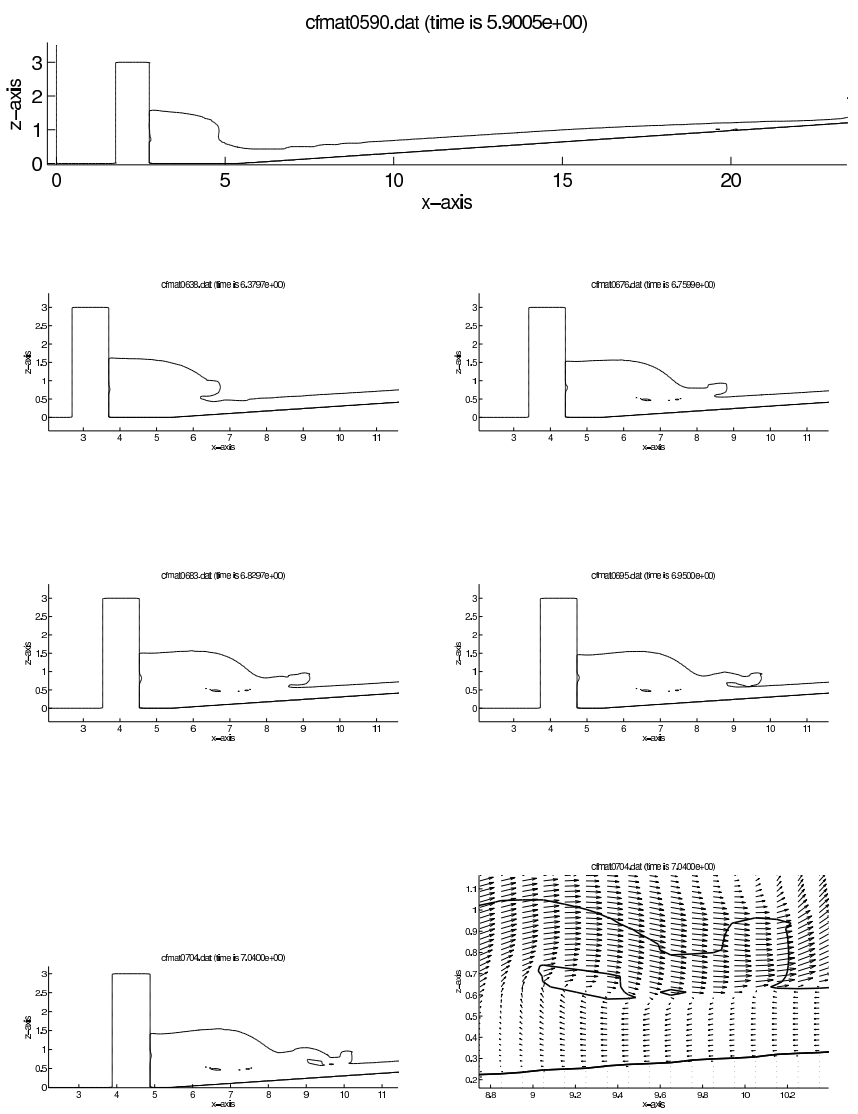

Figure 12. Snapshots of wave breaking for two-phase simulation and a mild 1:15 slope. The last snapshot shows the velocity field around the trapped air pocket at $t=7.04 \mathrm{~s}$.

\section{CONCLUSIONS}

The hydrodynamics of different offshore applications can be simulated numerically using an iVOF Navier-Stokes solver. This paper shows the results of the extension of the numerical model to a two-phase model.

The main flow variables are now calculated in all grid cells. Furthermore, the density is now calculated at all locations. Particular attention has been paid to the calculation of the density at the free surface. By doing this in the right way, spurious velocities are prevented.

To improve the simulation of the dynamics of the air phase, compressibility has been introduced. The air phase is subject to adiabatic compression and expansion.

For different test cases, it has been shown earlier that the simulation results from the two-phase model correlate better with available measurement results [10] than the results from the onephase model. In this paper, the two-phase model has been tested 
on breaking waves over a sloping bottom. Compared with onephase simulations, waves are moving forward slower in the twophase simulation due their effort to push away the viscous air. The bottom slope has also a pronounced effect on the wave breaking and the shape of the formed air pockets. The proper simulation of the dynamics of the trapped air pockets in the twophase model requires a fine grid, especially for "long" geometries with mild bottom slopes.

\section{ACKNOWLEDGMENT}

This research is supported by the Dutch Technology Foundation STW, applied science division of NWO and the technology programme of the Ministry of Economic Affairs.

\section{REFERENCES}

[1] Bullock, G.N., Crawford, A.R., Henson, P.J., Walkden, M.J.A., Bird, P.A.D. The influence of air and scale on wave impact pressures. Coastal Engineering, 42:291-312, 2001.

[2] Greco, M., Colicchio, G., Faltinsen, O.M. Application of a 2D BEM-level set domain decomposition to the greenwater problem. 20th International Workshop on Water Waves and Floating Bodies, Longyearbyen, Norway, 2005.

[3] Garzon, M., Adalsteinsson, D., Gray, L., Sethian, J.A. Wave breaking over sloping beaches using a coupled boundary integral-level set method. Lawrence Berkely National Laboratory, Univeristy of California, paper LBNL54480, 2003.

[4] Gerrits, J., Veldman, A.E.P. Dynamics of liquid-filled spacecraft. Journal of Engineering Mathematics, 45:21-38, 2003.

[5] Grilli, S.T., Subramanya, R. Numerical modeling of wave breaking induced by fixed or moving boundaries. Computational Mechanics, 17:374-391, 1996.

[6] Hirt, C.W., Nichols, B.D. Volume Of Fluid (VOF) Method for the Dynamics of Free Boundaries. Journal of Computational Physics, 39:201-225, 1981.

[7] Kleefsman, K.M.T., Fekken, G., Veldman, A.E.P., Buchner, B., Iwanowski, B. A Volume-Of-Fluid Based Simulation Method For Wave Impact problems. Journal of Computational Physics, 206:363-393, 2005.

[8] Lachaume, C., Biausser, B., Grilli, S.T., Fraunie, P., Guignard, S. Modeling of Breaking and Post-breaking Waves on Slopes by Coupling of BEM and VOF methods 13th International Offshore and Polar Engineering Conference, Hawaii, USA, 2003.

[9] Scardovelli, R., Zaleski, S. Direct Numerical Simulation of Free-Surface and Interfacial Flow. Annual Review of Fluid Mechanics, 31:567-603, 1999.

[10] Wemmenhove, R., Loots, G.E., Luppes, R., Veldman, A.E.P. Modeling two-phase flow with offshore applications. 24th Offshore Mechanics and Arctic Engineering Conference, paper 67460, Halkidiki, Greece, 2005.

[11] Yabe, T., Xiao, F., Utsumi, T. The constrained interpolation profile method for multiphase analysis. Journal of Computational Physics, 169:556-593, 2001. 